\title{
Mathematical Model for the Epidemiology of Fowl Pox Infection Transmission That Incorporates Discrete Delay
}

\author{
Udofia, Ekere Sunday ${ }^{1}$, Sampson, Marshal Imeh ${ }^{2}$ \\ ${ }^{I}$ Department of Mathematics/Statistics, Akwa Ibom State University, Mkpat Enin, Nigeria. \\ ${ }^{2}$ Department of Mathematics/Statistics, Akwa Ibom State University, Mkpat Enin, Nigeria.
}

\begin{abstract}
In this paper, we formulate the mathematical model for the epidemiology of fowl pox infection transmission that incorporates discrete delay. The model results in a discrete delay system of ordinary differential equations with delay parameter $\tau \geq 0$. Analysing the system using theorems from differential and integral calculus, we discovered that the number of infectives after a very long time from the day of inception of the epidemic is constant. $\frac{\left(\frac{1}{2} \delta-\alpha\right) N+\mu}{\frac{1}{4} \delta-\frac{1}{2} \alpha}$. The disease free equilibrium and the endemic equilibrium of the system were both established. Using the computable criteria for stability of discrete delay system, unlike ordinary differential equations models, we obtained exponential polynomial equations.

Analysing the system using lemmas and theorems from [1], [7], [4], it was established that both the disease free equilibrium endemic equilibrium points of the system (2.1) - (2.3) are stable in the absence of delay and unstable with increasing delay if $\quad \beta<\frac{\mu(\mu-\lambda)}{\alpha-\delta}$, that is, the rate at which the birds are recruited into the system must be greater than the rate at which birds die and the rate at which infection transmission is taking place.
\end{abstract}

Keywords: epidemiology, discrete delay, fowl pox, delay parameter, stability.

\section{Introduction}

Delay is used to mean "time lag" in the transmission process of an epidemic. This refers to the period taken for the infectious agent in the infected to develop. It is only after this period that the infected birds become infectious. In modelling biological and physical sciences, it is sometimes necessary to take into consideration the time delays inherent in the phenomena [5].

Fowl pox can be transmitted by direct or indirect contact. The virus is highly resistant in dried scabs and under certain conditions may survive for months on contaminated premises. The disease may be transmitted by a number of species of mosquitoes. Mosquitoes can harbour infective virus for a month or more after feeding on affected birds. After the infection is introduced, it spreads within the flock by mosquitoes as well as direct and indirect contact. Recovered birds do not remain carriers.

There are two main strains of the disease, the first appearing as greyish warty scabs on the comb, wattles and faces of the birds. They can result in serious disfigurement making such birds useless for showing. The second strain is more serious, causing cheesy substances to form in the respiratory passages, particularly the throat, which can cause death by asphyxiation [8]

There is no sure cure, other than treating the scabs with iodine and scraping the muck out of the birds' throats and painting with iodine. The bird needs to be removed kept in an uncrowded area. Clear the eyes and beaks so they can see to eat and drink on their own, use warm water with a very weak solution of iodine in the water.

The good news is that the birds naturally recover in 2 to 4 weeks usually, and are then immune to this particular strain of the disease. But some remain carriers and may become reinfected during molt and other times of stress. Fowl pox infection is classified among diseases in which individual birds can go into former state that is become ill after apparent recovery (relapse- recovery). [11]

Inyama, s. C(2006), in his work on the qualitative study of Kermack and Mckendrick's epidemic model, derived a special case of the model for recurrent disease( relapse-recovery model) with the assumption that after a fixed length of time an infective return to being fully susceptible again . Using the relapse-recovery model, he investigated the severity of the epidemic and discovered that the number of infectives after a very long time from the inception of the epidemic is constant. He also established the trajectories that help to know the extent of the severity. Through these trajectories, it was shown that the severity of this epidemic can be estimated when the rate of infectiousness and removal rate are estimated.

Obabiyi, O. S (2009), in his research on discrete delay-parameter epidemiological model, established a cyclic delay parameter epidemiological model for a closed population which is divided into susceptible and 
infective class. He established that the delay does not change the nature of asymptotic stability of endemic steady state whenever one uses Lyapunov approach.

In this paper, we present a mathematical model for the transmission dynamic of fowl pox considering the delay inherent in the process of transmission. That is, it takes a period of time say, $\tau$ for the infectious agent to develop and become infectious. Also after some time when the bird has recovered from one strain of the fowl pox may become susceptible to the second strain of fowl pox infection.

\subsection{The Assumption}

\section{The Model Framework}

Delay is positive that is, $\tau \geq 0$

Infected birds are infectious after a time-lag $\tau$

Recovered birds relapse into its former state after a time-lag $\tau$

The total population of birds is divided into the susceptible (S), infected (I) and the removed birds (R).

\subsection{Parameters/ Symbol}

$\mathrm{S}(\mathrm{t})=$ susceptible birds at time $\mathrm{t}$

$I(t)=$ infected birds at time $t$

$\mathrm{R}(\mathrm{t})=$ removed birds at time $\mathrm{t}$

$\beta=$ recruitment rate of birds

$\alpha=$ the rate of infection

$\delta=$ the rate at which birds recover and join the susceptible population

$\mu \quad=$ natural death rate of birds

$\tau=$ delay parameter

$\lambda=$ removal rate of the infection

We divide the population $(\mathrm{N})$ of birds into three sub-populations according to their states at time $\mathrm{t}>0$; Susceptible (S), Infected (I), and Removed(R). Our model describes the transmission dynamics of fowl pox infection based on two strains. Here we consider that birds which recover from one can become susceptible to the other strain. The infected birds do not become infectious instantly; the infectious agent develops in the infected over a certain period of time. The delay parameter $\tau$ denotes the time interval taken for the infected to become infectious or the recovered bird to become susceptible.

The susceptible population is increased by recruitment of birds (either by birth or immigration) and birds which recover and become susceptible. This population is reduced by infection and natural death. The infected population is increased by infection of the susceptible. It is reduced by those that become susceptible after recovery and natural death. The population of the removed bird is increased by removal from the infected and reduce by natural death

\subsection{The Model Equations}

$$
\begin{aligned}
& \frac{\mathrm{dS}}{\mathrm{dt}}=\beta \mathrm{S}(\mathrm{t})-\alpha \mathrm{S}(\mathrm{t}) \mathrm{I}(\mathrm{t}-\tau)+\delta \mathrm{S}(\mathrm{t}-\tau) \mathrm{I}(\mathrm{t}-\tau)-\mu \mathrm{S} \\
& \frac{\mathrm{dI}}{\mathrm{dt}}=\alpha \mathrm{S}(\mathrm{t}) \mathrm{I}(\mathrm{t}-\tau)-\delta \mathrm{S}(\mathrm{t}-\tau) \mathrm{I}(\mathrm{t}-\tau)-\lambda \mathrm{I}(\mathrm{t}-\tau)-\mu \mathrm{I} \\
& \frac{\mathrm{dR}}{\mathrm{dt}}=\lambda \mathrm{I}(\mathrm{t}-\tau)-\mu \mathrm{R}
\end{aligned}
$$

\section{Severity of the Epidemic}

We consider that the removed class does have impact on the severity of the epidemic. The model becomes

$$
\begin{aligned}
& \frac{\mathrm{d} S}{\mathrm{dt}}=\beta \mathrm{S}(\mathrm{t})-\alpha \mathrm{S}(\mathrm{t}) \mathrm{I}(\mathrm{t}-\tau)+\delta \mathrm{S}(\mathrm{t}-\tau) \mathrm{I}(\mathrm{t}-\tau)-\mu \mathrm{S}(\mathrm{t}) \\
& \frac{\mathrm{d} \mathrm{I}}{\mathrm{dt}}=\alpha \mathrm{S}(\mathrm{t}) \mathrm{I}(\mathrm{t}-\tau)-\delta \mathrm{S}(\mathrm{t}-\tau) \mathrm{I}(\mathrm{t}-\tau)-\mu \mathrm{I}(\mathrm{t}) \\
& \text { Since } \mathrm{I}+\mathrm{S}=\mathrm{N}, \quad \Rightarrow \mathrm{S}=\mathrm{N}-\mathrm{I}
\end{aligned}
$$

Hence (3.2) becomes

$\frac{\mathrm{dI}}{\mathrm{dt}}=\alpha[\mathrm{N}-\mathrm{I}(\mathrm{t})] \mathrm{I}(\mathrm{t}-\tau)-\delta[\mathrm{N}-\mathrm{I}(\mathrm{t}-\tau)] \mathrm{I}(\mathrm{t}-\tau)-\mu \mathrm{I}(\mathrm{t})$

Here we seek to determine the number of the infectives after a long time $(\mathrm{t} \rightarrow \infty)$. We then put this in a theorem 


\section{Theorem 3.1}

Let $\frac{d \mathrm{I}}{\mathrm{dt}}=\alpha[\mathrm{N}-\mathrm{I}(\mathrm{t})] \mathrm{I}(\mathrm{t}-\tau)-\mu[\mathrm{N}-\mathrm{I}(\mathrm{t}-\tau)] \mathrm{I}(\mathrm{t}-\tau)-\mu_{2} \mathrm{I}(\mathrm{t})$

be a discrete delay system. Given that $I(t-\tau)=\frac{1}{2} I(t)$ then there exist a limit of $I(t)$ (a constant value $I^{*}$ ) as $t$ tends to infinity.

Proof:

Let $I(t-\tau)=\frac{1}{2} I(t)$

Then $\frac{\mathrm{dI}}{\mathrm{dt}}=\alpha[\mathrm{N}-\mathrm{I}(\mathrm{t})] \frac{1}{2} \mathrm{I}(\mathrm{t})-\delta\left[\mathrm{N}-\frac{1}{2} \mathrm{I}(\mathrm{t})\right] \frac{1}{2} \mathrm{I}(\mathrm{t})-\mu \mathrm{I}(\mathrm{t})$

Let $\frac{1}{2} \alpha=a$ and $\frac{1}{2} \delta=b$

Then $\frac{\mathrm{dI}}{\mathrm{dt}}=\mathrm{aI}[\mathrm{N}-\mathrm{I}(\mathrm{t})]-\mathrm{bI}(\mathrm{t})\left[\mathrm{N}-\frac{1}{2} \mathrm{I}(\mathrm{t})\right]-\mu \mathrm{I}(\mathrm{t})$

$\frac{\mathrm{dI}}{\mathrm{dt}}=(\alpha \mathrm{N}-\mathrm{bN}-\mu) \mathrm{I}+\left(\frac{1}{2} \mathrm{~b}-\mathrm{a}\right) \mathrm{I}^{2}$ which implies that $\frac{\mathrm{dI}}{\mathrm{I}(\mathrm{K}+\mathrm{DI})}=\mathrm{dt}$

Where $\mathrm{K}=\alpha \mathrm{N}-\mathrm{bN}-\mu$ and $\mathrm{D}=\frac{1}{2} \mathrm{~b}-\mathrm{a}$

Using partial fractions in the L. H. S and simplifying gives $\frac{\mathrm{dI}}{\mathrm{KI}}-\frac{\mathrm{Ddt}}{\mathrm{K}(\mathrm{K}+\mathrm{DI})}=\mathrm{dt}$

Integrating gives $\frac{\mathrm{I}}{\mathrm{K}+\mathrm{DI}}=\rho \mathrm{e}^{\mathrm{Kt}}$

Applying the initial boundary condition $\mathrm{I}(0)=\mathrm{I}_{0}$, we have $\frac{\mathrm{I}_{0}}{\mathrm{~K}+\mathrm{DI}_{0}}=\rho$

$\Rightarrow \frac{\mathrm{I}}{\mathrm{K}+\mathrm{DI}}=\frac{\mathrm{I}_{0}}{\mathrm{~K}+\mathrm{DI}_{0}} \mathrm{e}^{\mathrm{Kt}}$

Solving for I gives $\quad \mathrm{I}=\frac{\mathrm{KI}_{0}}{\mathrm{~K}+\mathrm{DI}_{0}-\mathrm{DI}_{0} \mathrm{e}^{\mathrm{Kt}}}$

$\mathrm{I}=\frac{\mathrm{KI}_{0}}{\left(\mathrm{~K}+\mathrm{DI}_{0}\right) \mathrm{e}^{-\mathrm{Kt}}-\mathrm{DI}_{0}}$

As $t \rightarrow \infty, \mathrm{I} \rightarrow \mathrm{I}^{*}$ where $\mathrm{I}^{*}=\frac{\left(\frac{1}{2} \delta-\alpha\right) \mathrm{N}+\mu}{\frac{1}{4} \delta-\frac{1}{2} \alpha}$

which is a constant. Hence, the number of infectives after a very long time from the day of inception of the epidemic is constant.

\section{Stability Of The Equilibrium State}

Considering, (3.1 and 3.2), the disease free equilibrium point is given as

$\mathrm{E}_{0}=\left(\mathrm{S}^{*}, \mathrm{I}^{*}\right)=\left(\frac{\mu_{2}}{\beta}, 0\right)$

The endemic equilibrium point is given as $E_{E}=\left(S^{*}, I^{*}\right)=\left(\frac{\mu_{2}}{\alpha-\mu}, \frac{\beta-\mu_{2}}{\alpha-\mu}\right)$

Using the theory of computable criteria for stability of discrete delay system, Obabiyi, 2009, Iheagwam, 2006, Forde, 2005.

$\frac{\mathrm{dx}}{\mathrm{dt}}=\sum_{\mathrm{i}=0}^{\mathrm{n}} \mathrm{A}_{\mathrm{i}}(\mathrm{t}) \mathrm{x}\left(\mathrm{t}-\mathrm{h}_{\mathrm{i}}\right)$

$A_{i}$ is the matrix of the discrete delay system, $h_{i}$ is the discrete delay.

Then the characteristic polynomial of the system is given as

$\mathrm{F}(\lambda)=\left|\lambda \mathrm{I}-\sum_{\mathrm{i}=0}^{\mathrm{n}} \mathrm{A}_{\mathrm{i}} \mathrm{e}^{-\lambda \mathrm{h}_{\mathrm{i}}}\right|$

Theorem 4.7: (condition for exponential asymptotic stability of discrete delay system)

If the roots $\lambda$ of the characteristic equation (4.2) have negative real parts, there exist constants $\alpha>0, k>0$ such that the solution of (4.13) satisfies

$\|\mathrm{x}(\mathrm{t})\| \leq\|\varnothing\| \mathrm{e}^{-\alpha\left(\mathrm{t}-\mathrm{t}_{0}\right)}, \mathrm{t} \geq \quad 0 \quad, \varnothing \in \mathrm{C} \quad$ (Forde, 2005.)

where $C$ is a space of continuous function

From the systems (3.1-3.2) and (4.2)

$$
\mathrm{A}_{0}=\left(\begin{array}{cc}
\beta-\mu_{2}-\alpha \mathrm{I}^{*} & 0 \\
\alpha \mathrm{I}^{*} & -\mu_{2}
\end{array}\right) \mathrm{A}_{1}=\left(\begin{array}{cc}
\mu \mathrm{I}^{*} & (\mu-\alpha) \mathrm{S}^{*} \\
-\mu \mathrm{I}^{*} & (\alpha-\mu) \mathrm{S}^{*}
\end{array}\right)
$$


Considering the disease free equilibrium points $\mathrm{E}_{0}=\left(\mathrm{S}^{*}, \mathrm{I}^{*}\right)=\left(\frac{\mu_{2}}{\beta}, 0\right)$

$$
A_{0}=\left(\begin{array}{cc}
\beta-\mu_{2}- & 0 \\
0 & -\mu_{2}
\end{array}\right), A_{1}=\left(\begin{array}{cc}
0 & (\mu-\alpha) \frac{\mu_{2}}{\beta} \\
0 & (\alpha-\mu) \frac{\mu_{2}}{\beta}
\end{array}\right)
$$

Using the computable criteria for stability of discrete delay system (4.1) and (4.2), just as for ordinary differential equations, one seeks exponential solutions, and computes a characteristic equation.

$\mathrm{f}(\lambda)=\left|\left(\begin{array}{cc}\lambda & 0 \\ 0 & \lambda\end{array}\right)-\left[\left(\begin{array}{cc}\beta-\mu_{2} & 0 \\ 0 & \mu_{2}\end{array}\right)+\left(\begin{array}{cc}0 & (\mu-\alpha) \frac{\mu_{2}}{\beta} \\ 0 & (\alpha-\mu) \frac{\mu_{2}}{\beta}\end{array}\right) \mathrm{e}^{-\lambda \tau}\right]\right|=0$

Rather than a polynomial equation, we arrive at a transcendental equation of the form

$$
\lambda^{2}-\beta \lambda+\mu_{2}\left(\beta-\mu_{2}\right)+\left[(\mu-\alpha) \frac{\mu_{2}}{\beta} \lambda+\left(\mu_{2}-\beta\right)(\mu-\alpha) \frac{\mu_{2}}{\beta}\right] \mathrm{e}^{-\lambda \tau}=0 \ldots \ldots
$$

Lemma (4.1) Cooke and Grossman, 1982. Consider the equation

$\lambda^{2}+a \lambda+b+(c \lambda+d) e^{-\lambda \tau}=0$

Where $a, b, c$, and $d$ are real numbers. Let the hypothesis

$\mathrm{H}_{1}: \mathrm{a}+\mathrm{c}>0$

$\mathrm{H}_{2}: \mathrm{b}+\mathrm{d}>0$

$\mathrm{H}_{3}: \mathrm{c}^{2}-\mathrm{a}^{2}+2 \mathrm{~b}<0$ and $\mathrm{b}^{2}-\mathrm{d}^{2}>0$ or $\left(\mathrm{c}^{2}-\mathrm{a}^{2}+2 \mathrm{e}\right)^{2}<4\left(\mathrm{~b}^{2}-\mathrm{d}^{2}\right)$

$\mathrm{H}_{4}: \mathrm{b}^{2}-\mathrm{d}^{2}<0$ or $\mathrm{c}^{2}-\mathrm{a}^{2}+2 \mathrm{~b}>0$ and $\left(\mathrm{c}^{2}-\mathrm{a}^{2}+2 \mathrm{~b}\right)^{2}=4\left(\mathrm{~b}^{2}-\mathrm{d}^{2}\right)$

(i) If $\left(\mathrm{H}_{1}\right)-\left(\mathrm{H}_{3}\right)$ hold, then all roots of equation (4.5) have negative real parts for all $\tau \geq 0$

(ii) If $\mathrm{H}_{1}, \mathrm{H}_{2}$, and $\mathrm{H}_{4}$ hold, then there exist $\tau_{0}>0$ such that when $\tau \in\left[0 . \tau_{0}\right)$ all roots of equation (4.5) have negative real parts, and when $\tau=\tau_{0}$ has a pair of purely imaginary roots $\pm \xi_{+}$, and when $\tau>\tau_{0}$ equation (4.5) has at least one root with positive real part. Where

$$
\begin{aligned}
& \tau_{0}=\frac{1}{\xi_{+}} \arccos \left(\frac{\mathrm{d}\left(\xi_{+}^{2}-\mathrm{b}\right)-\mathrm{ac} \xi_{+}^{2}}{\mathrm{c}^{2} \xi_{+}^{2}+\mathrm{d}^{2}}\right\} \\
& \xi_{+}^{2}=\frac{1}{2}\left(\mathrm{c}^{2}-\mathrm{a}^{2}+2 \mathrm{e}\right) \pm \frac{1}{2}\left[\left(\mathrm{c}^{2}-\mathrm{a}^{2}+2 \mathrm{~b}\right)^{2}-4\left(\mathrm{~b}^{2}-\mathrm{d}^{2}\right)\right]^{\frac{1}{2}} \\
& \text { Hence } \mathrm{a}=-\beta \quad, \mathrm{b}=\mu_{2}\left(\beta-\mu_{2}\right), \mathrm{c}=(\mu-\alpha) \frac{\mu_{2}}{\beta}, \mathrm{d}=\left(\mu_{2}-\beta\right)(\mu-\alpha) \frac{\mu_{2}}{\beta}
\end{aligned}
$$

The conditions for the roots of (4.4) to have negative real parts are

$\mu_{2}(\mu-\alpha)>\beta^{2}, \quad \beta>\mu-\alpha, \quad \mu_{2}^{2}(\mu-\alpha)^{2}>\beta^{2}\left(2 \mu_{2}-2 \mu_{2} \beta+\beta^{2}\right.$ and $\beta>\mu-\alpha$

The disease free equilibrium point of the system is asymptotically stable if, and only if, all the roots of the characteristic polynomial possess negative real parts. Furthermore, the disease free equilibrium point of the system is asymptotically stable if, and only if, it is exponentially asymptotically stable, Hong-shan and Hongyan, 2002.

Hence the disease free equilibrium is exponentially asymptotically stable for $\tau \geq 0$ if $\mu_{2}(\mu-\alpha)>\beta^{2}$, $\beta>\mu-\alpha, \quad \mu_{2}^{2}(\mu-\alpha)^{2}>\beta^{2}\left(2 \mu_{2}-2 \mu_{2} \beta+\beta^{2}\right.$ and $\beta>\mu-\alpha$

Lemma 4.2 Cooke and Grossman, 1982: A steady state with characteristic equation

$\lambda^{2}+a \lambda+b+(c \lambda+d) e^{-\lambda \tau}=0$

is stable in the absence of delay and becomes unstable with increasing delay if and only if

(i) $\mathrm{a}+\mathrm{c}>0$ and $b+d>0$

(ii) Either $b^{2}<d^{2}$ or $b^{2}>d^{2}, a^{2}<c^{2}+2 b$

Comparing (4.4) with (4.6), then we have that

$$
\text { and }\left(a^{2}-c^{2}-2 b\right)^{2}>4\left(b^{2}-d^{2}\right)
$$

$\mathrm{a}=\mu_{2}-\beta, \mathrm{b}=0,=\left(\mu_{2}+\alpha-\mu\right) \frac{\mu_{2}}{\beta}, \mathrm{d}=\left(\beta-\mu_{2}\right)\left(\mu_{2}+\alpha-\mu\right) \frac{\mu_{2}}{\beta}$

Hence the conditions for the disease free equilibrium of the system (3.1) and (3.2) to be stable in the absence of delay and unstable in the presence of increasing delay are as follows:

$\mu_{2} \beta-\beta^{2}-\mu_{2}^{2}-\alpha \mu_{2}+\mu \mu_{2}<0$ and $\mu_{2}+\alpha>\mu$ and

$$
\mu_{2}-\beta-\left(\left(\mu_{2}+\alpha-\mu\right) \frac{\mu_{2}}{\beta}\right)^{2}+4\left(\left(\beta-\mu_{2}\right)\left(\mu_{2}+\alpha-\mu\right) \frac{\mu_{2}}{\beta}\right)^{2}>0
$$

For the endemic equilibrium $E_{E}=\left(S^{*}, I^{*}\right)=\left(\frac{\mu_{2}}{\alpha-\mu}, \frac{\beta-\mu_{2}}{\alpha-\mu}\right)$ 
$\mathrm{A}_{0}=\left(\begin{array}{cc}\mu \gamma & 0 \\ \alpha \gamma & -\mu_{2}\end{array}\right), \mathrm{A}_{1}=\left(\begin{array}{cc}\mu \gamma & -\mu_{2} \\ -\mu \gamma & \mu_{2}\end{array}\right)$
Where $\gamma=\frac{\beta-\mu_{2}}{\alpha-\mu}$

Using the computable criteria for stability of discrete delay system (4.1) and (4.2), just as for ordinary differential equations, one seeks exponential solutions, and computes a characteristic equation.

$\mathrm{f}(\lambda)=\left|\left(\begin{array}{cc}\lambda & 0 \\ 0 & \lambda\end{array}\right)-\left[\left(\begin{array}{cc}\mu \gamma & 0 \\ \alpha \gamma & -\mu_{2}\end{array}\right)+\left(\begin{array}{cc}\mu \gamma & -\mu_{2} \\ -\mu \gamma & \mu_{2}\end{array}\right) \mathrm{e}^{-\lambda \tau}\right]\right|=0$

Rather than a polynomial equation, we arrive at a transcendental equation of the form

$\lambda^{2}+\left(\mu_{2}-\mu \gamma\right) \lambda+\gamma \mu_{2}(\alpha-\mu)+\left[\left(\mu \gamma-\mu_{2}\right) \lambda+2 \mu \gamma \mu_{2}\right] \mathrm{e}^{-\lambda \tau}-2 \mu \mu_{2} \gamma \mathrm{e}^{-2 \lambda \tau}=0 \quad$ (4.7)

Therefore using the result of Lemma4.1, we have $a=\mu_{2}-\mu \gamma b=\gamma \mu_{2}(\alpha-\mu), c=\mu \gamma-\mu_{2}$ and $\mathrm{d}=2 \mu \gamma \mu_{2}$

On careful observation and analysis, we discover that condition $\left(\mathrm{H}_{1}\right)$ does not hold for the endemic equilibrium. Hence, at the endemic equilibrium the system is unstable.

Also using the result of lemma 4.2: $\left(a^{2}-c^{2}-2 b\right)^{2}>4\left(b^{2}-d^{2}\right)$ and $b^{2}<d^{2}$ given that $a=\mu_{2}-$ $\mu \gamma \mathrm{b}=\gamma \mu_{2}(\alpha-\mu), \mathrm{c}=\mu \gamma-\mu_{2}$ and $\mathrm{d}=2 \mu \gamma \mu_{2}$.

\section{Result:}

We obtain the condition for (4.7) to have negative real parts as $\beta>\mu_{2}$ and $\alpha<3 \mu$. That is the rate at which the susceptible birds are recruited into the poultry must be greater than the rate the birds die naturally. And the rate at which the birds are becoming infectious must be less than the rate at which they recover and join the susceptible population.

Hence the system is stable in the absence of delay and becomes unstable with increasing delay

\section{The Model With Removal Class}

The model with removed class as shown in (2.1-2.3)

$$
\begin{gathered}
\frac{d S}{d t}=\beta S(t)-\alpha S(t) I(t-\tau)+\mu S(t-\tau) I(t-\tau)-\mu_{2} S \\
\frac{d I}{d t}=\alpha S(t) I(t-\tau)-\mu S(t-\tau) I(t-\tau)-\lambda I(t-\tau)-\mu_{2} I \\
\frac{d R}{d t}=\lambda I(t-\tau)-\mu_{2} R
\end{gathered}
$$

\subsection{Stability Of The Equilibrium State}

In studying a delay model, linearization of the system at its steady state gives a transcendental characteristics polynomial equation. It is well known that the steady state is stable if all eigenvalues of the exponential polynomial equation have negative real parts, and unstable if at least one root has a positive real part. However, there is strong possibility that if the coefficients of the exponential polynomial satisfy certain assumption, the real parts of all eigenvalue remains negative for all values of the delay, Shigui and Junjie, 2001 The disease Free equilibrium point is given as $\mathrm{E}_{0}=\left(\mathrm{S}^{*}, \mathrm{I}^{*} \mathrm{R}^{*}\right)=\left(\frac{\mu_{2}}{\beta}, 0,0\right)$

The endemic equilibrium point is given as

$$
\begin{aligned}
\mathrm{E}_{\mathrm{E}}=\left(\mathrm{S}^{*}, \mathrm{I}^{*}, \mathrm{R}^{*}\right) & =\left(\frac{\mu_{2}+\lambda}{\alpha-\mu}, \frac{\beta-\mu_{2}}{\alpha-\mu}, \frac{\lambda\left(\mu_{2}-\beta\right)}{\mu_{2}(\alpha-\mu)}\right) \\
\mathrm{A}_{0} & =\left(\begin{array}{ccc}
\beta-\mu_{2}-\alpha \mathrm{I}^{*} & 0 & 0 \\
\alpha \mathrm{I}^{*} & -\mu_{2} & 0 \\
0 & 0 & -\mu_{2}
\end{array}\right), \mathrm{A}_{1}=\left(\begin{array}{ccc}
\mu \mathrm{I}^{*} & (\mu-\alpha) \mathrm{S}^{*} & 0 \\
-\mu \mathrm{I}^{*} & (\alpha-\mu) \mathrm{S}^{*} & 0 \\
0 & \lambda & 0
\end{array}\right)
\end{aligned}
$$

Considering the disease free equilibrium $\mathrm{E}_{0}=\left(\mathrm{S}^{*}, \mathrm{I}^{*} \mathrm{R}^{*}\right)=\left(\frac{\mu_{2}}{\beta}, 0,0\right)$

$$
A_{0}=\left(\begin{array}{ccc}
\beta-\mu_{2} & 0 & 0 \\
0 & -\mu_{2} & 0 \\
0 & 0 & -\mu_{2}
\end{array}\right), A_{1}=\left(\begin{array}{ccc}
0 & (\mu-\alpha) \frac{\mu_{2}}{\beta} & 0 \\
0 & (\alpha-\mu) \frac{\mu_{2}}{\beta} & 0 \\
0 & \lambda & 0
\end{array}\right)
$$

Using the computable criteria for stability of discrete delay system (4.1) and (4.2), just as for ordinary differential equations, one seeks exponential solutions, and computes a characteristic equation. 
$f(X)=\left|\left(\begin{array}{ccc}X & 0 & 0 \\ 0 & X & 0 \\ 0 & 0 & X\end{array}\right)-\left[\left(\begin{array}{ccc}\beta-\mu_{2} & 0 & 0 \\ 0 & -\mu_{2} & 0 \\ 0 & 0 & -\mu_{2}\end{array}\right)+\left(\begin{array}{ccc}0 & (\mu-\alpha) \frac{\mu_{2}}{\beta} & 0 \\ 0 & (\alpha-\mu) \frac{\mu_{2}}{\beta} & 0 \\ 0 & \lambda & 0\end{array}\right) e^{-X \tau}\right]\right|=0$

$$
\Rightarrow\left|\begin{array}{ccc}
\mathrm{X}-\left(\beta-\mu_{2}\right) & -(\mu-\alpha) \frac{\mu_{2}}{\beta} \mathrm{e}^{-\mathrm{X \tau}} & 0 \\
0 & \mathrm{X}+\mu_{2}-(\alpha-\mu) \frac{\mu_{2}}{\beta} \mathrm{e}^{-\mathrm{X \tau}} & 0 \\
0 & -\lambda \mathrm{e}^{-\mathrm{X \tau}} & \mathrm{X}+\mu_{2}
\end{array}\right|=0
$$

Rather than a polynomial equation, we arrive at a transcendental equation of the form

$\mathrm{X}^{3}+\left(2 \mu_{2}-\beta+\mu\right) \mathrm{X}^{2}-(\alpha-\mu) \frac{\mu_{2}}{\beta} \mathrm{e}^{-\mathrm{X} \tau} \mathrm{X}^{2}+\left(\mu_{2}^{2}-2 \mu_{2}(\beta-\mu)\right) \mathrm{X}+(\alpha-\mu)\left(\beta-\mu-\mu_{2}\right) \frac{\mu_{2}}{\beta} \mathrm{e}^{-\mathrm{X} \tau} \mathrm{X}-$

$(\beta-\mu) \mu_{2}^{2}+(\beta-\mu)(\alpha-\mu) \frac{\mu_{2}^{2}}{\beta} \mathrm{e}^{-X \tau}=0$

Lemma 5.1. (Shigui and Junjie, 2001).

Consider the equation

$\mathrm{X}^{3}+\mathrm{aX}^{2}+\mathrm{bX}+\mathrm{c}+\mathrm{de}^{-\mathrm{X \tau}}=0$

Suppose $a>0, c+d>0, a b-c-d>0$

(i) If $c^{2}-d^{2} \geq 0$ and $p^{2}-3 q>0$, then the roots of equation (5.1) have negative real parts for all $\tau \geq 0$

Rearranging (5.1), we have

$$
\mathrm{q}=\mathrm{b}^{2}-2 \mathrm{ac} \text { and } \mathrm{r}=\mathrm{c}^{2}-\mathrm{d}^{2}
$$

$$
\begin{aligned}
\mathrm{X}^{3}+\left(2 \mu_{2}-\beta\right. & +\mu) \mathrm{X}^{2}+\left(\mu_{2}^{2}-2 \mu_{2}(\beta-\mu)\right) \mathrm{X}+(\mu-\beta) \mu_{2}^{2}+(\beta-\mu)(\alpha-\mu) \frac{\mu_{2}^{2}}{\beta} \mathrm{e}^{-\mathrm{X} \tau}-(\alpha-\mu) \frac{\mu_{2}}{\beta} \mathrm{e}^{-\mathrm{X} \tau} \mathrm{X}^{2} \\
& +(\alpha-\mu)\left(\beta-\mu-\mu_{2}\right) \frac{\mu_{2}}{\beta} \mathrm{e}^{-\mathrm{X} \tau} \mathrm{X}=0
\end{aligned}
$$

Using the result of Lemma (5.1), we have that

$a=\left(2 \mu_{2}-\beta+\mu\right), b=\left(\mu_{2}^{2}-2 \mu_{2}(\beta-\mu)\right) c=(\mu-\beta) \mu_{2}^{2} \quad, d=(\beta-\mu)(\alpha-\mu) \frac{\mu_{2}^{2}}{\beta}$

For the roots of (5.1) to have negative real parts then $c+d>0, c^{2}-d^{2} \geq 0$, that is $(\mu-\beta) \mu_{2}^{2}+$ $(\beta-\mu)(\alpha-\mu) \frac{\mu_{2}^{2}}{\beta}>0$ and $\left((\mu-\beta) \mu_{2}^{2}\right)^{2}-\left((\beta-\mu)(\alpha-\mu) \frac{\mu_{2}^{2}}{\beta}\right)^{2} \geq 0$

Simplifying, we have $\beta>\alpha-\mu$ and $\beta \geq \alpha-\mu$

Hence, at the disease free equilibrium $\mathrm{E}_{0}=\left(\mathrm{S}^{*}, \mathrm{I}^{*} \mathrm{R}^{*}\right)=\left(\frac{\mu_{2}}{\beta}, 0,0\right)$ the system is exponentially asymptotically stable if $\beta>\alpha-\mu$ and $\beta \geq \alpha-\mu$. That is the recruitment rate of the susceptible birds must be greater than the transmission rate of infection of birds in the poultry.

Furthermore; we use the method of Theorem 2.4 in [Forde, (2005)], which provides a general algorithm for determining stability of model with delay differential equations to determine the stability of (2.12.3).

Theorem 4.8: A steady state with characteristic equation

$\mathrm{X}^{3}+\mathrm{a}_{2} \mathrm{X}^{2}+\mathrm{a}_{1} \mathrm{X}+\mathrm{a}_{0}+\left(\mathrm{b}_{2} \mathrm{X}^{2}+\mathrm{b}_{1} \mathrm{X}+\mathrm{b}_{0}\right) \mathrm{e}^{-\mathrm{X \tau}}=0$

$$
\mathrm{A}=\mathrm{a}_{2}^{2}-\mathrm{b}_{2}^{2}-2 \mathrm{a}_{1}, \quad \mathrm{~B}=\mathrm{a}_{1}^{2}-\mathrm{b}_{1}^{2}+2 \mathrm{~b}_{2} \mathrm{~b}_{0}-2 \mathrm{a}_{2} \mathrm{a}_{0} \text { and } \mathrm{C}=\mathrm{a}_{0}^{2}-\mathrm{b}_{0}^{2}
$$

is stable in the absence of delay and becomes unstable with increasing delay if and only if $\mathrm{A}, \mathrm{B}$, and $\mathrm{C}$ are not all positive and

(i) $\quad \mathrm{a}_{2}+\mathrm{b}_{2}>0, \quad \mathrm{a}_{0}+\mathrm{b}_{0}>0,\left(\mathrm{a}_{2}+\mathrm{b}_{2}\right)\left(\mathrm{a}_{1}+\mathrm{b}_{1}\right)-\left(\mathrm{a}_{0}+\mathrm{b}_{0}\right)>0$

(ii) Either $\mathrm{C}<0$ or $\mathrm{C}>0, \mathrm{~A}^{2}-3 \mathrm{~B}>0$

(5.1) can be written in form of (5.3) as

$X^{3}+\left(2 \mu_{2}-\beta-\mu\right) X^{2}+\left(\mu_{2}^{2}-2(\beta-\mu)\right) X+(\mu-\beta) \mu_{2}^{2}+\left((\mu-\alpha) \frac{\mu_{2}}{\beta} X^{2}+(\alpha-\mu) \frac{\mu_{2}}{\beta}\left(\beta-\mu-\mu_{2}\right) X+\right.$

$\beta-\mu \alpha-\mu \mu 22 \beta e-X \tau=0$

$a_{2}=2 \mu_{2}-\beta-\mu, a_{1}=\mu_{2}^{2}-2(\beta-\mu) \quad, a_{0}=(\mu-\beta) \mu_{2}^{2} \quad, b_{2}=(\mu-\alpha) \frac{\mu_{2}}{\beta}$, 
$b_{1}=(\alpha-\mu) \frac{\mu_{2}}{\beta}\left(\beta-\mu-\mu_{2}\right), b_{0}=(\beta-\mu)(\alpha-\mu) \frac{\mu_{2}^{2}}{\beta}$

Analysing the characteristic equation (5.4) of the system (2.1-2.3) based on Theorem 4.8, we discovered that the system is stable in the absence of delay and unstable with increasing delay if $\mathrm{a}_{2}+\mathrm{b}_{2}>$ $0, \Rightarrow 2 \mu_{2}-\beta-\mu+(\mu-\alpha) \frac{\mu_{2}}{\beta}>0 \Rightarrow 2 \mu_{2}-\beta^{2}+\mu \mu_{2}-\alpha \mu_{2}>0$

$\Rightarrow \beta\left(2 \mu_{2}-\beta\right)>\mu_{2}(\alpha-\mu)$ and

$\mathrm{C}>0$, that is, $\mathrm{a}_{0}^{2}-\mathrm{b}_{0}^{2}>0 \Rightarrow\left((\mu-\beta) \mu_{2}^{2}\right)^{2}>\left((\beta-\mu)(\alpha-\mu) \frac{\mu_{2}^{2}}{\beta}\right)^{2}$

$\Rightarrow \beta^{2}>(\alpha-\mu)^{2} \Rightarrow \beta>\alpha-\mu$, that is the rate at which the birds are recruited into the system must be greater than the rate at which infection transmission is taking place.

Considering the disease endemic equilibrium

$\mathrm{E}_{\mathrm{E}}=\left(\mathrm{S}^{*}, \mathrm{I}^{*} \mathrm{R}^{*}\right)=\left(\frac{\mu_{2}+\lambda}{\alpha-\mu}, \frac{\mu_{2}-\beta}{\alpha-\mu}, \frac{\lambda(\mu-\beta)}{\mu_{2}(\alpha-\mu)}\right)$

$A_{0}=\left(\begin{array}{ccc}\beta-\mu_{2}-\alpha \frac{\mu_{2}-\beta}{\alpha-\mu} & 0 & 0 \\ \alpha \frac{\mu_{2}-\beta}{\alpha-\mu} & -\mu_{2} & 0 \\ 0 & 0 & -\mu_{2}\end{array}\right)^{\prime} A_{1}=\left(\begin{array}{ccc}\mu \frac{\mu_{2}-\beta}{\alpha-\mu} & (\mu-\alpha) \frac{\mu_{2}+\lambda}{\alpha-\mu} & 0 \\ -\mu \frac{\mu_{2}-\beta}{\alpha-\mu} & (\alpha-\mu) \frac{\mu_{2}+\lambda}{\alpha-\mu} & 0 \\ 0 & \lambda & 0\end{array}\right)$

Using the computable criteria for stability of discrete delay system (4.1) and (4.2), just as for ordinary differential equations, one seeks exponential solutions, and computes a characteristic equation.

$\mathrm{f}(\mathrm{X})$

$$
\begin{aligned}
& =\mid\left(\begin{array}{lll}
\mathrm{X} & 0 & 0 \\
0 & \mathrm{X} & 0 \\
0 & 0 & \mathrm{X}
\end{array}\right)-\left[\left(\begin{array}{ccc}
\beta-\mu_{2}-\alpha \frac{\mu_{2}-\beta}{\alpha-\mu} & 0 & 0 \\
\alpha \frac{\mu_{2}-\beta}{\alpha-\mu} & -\mu_{2} & 0 \\
0 & 0 & -\mu_{2}
\end{array}\right)+\left(\begin{array}{ccc}
\mu \frac{\mu_{2}-\beta}{\alpha-\mu} & (\mu-\alpha) \frac{\mu_{2}+\lambda}{\alpha-\mu} & 0 \\
-\mu \frac{\mu_{2}-\beta}{\alpha-\mu} & (\alpha-\mu) \frac{\mu_{2}+\lambda}{\alpha-\mu} & 0 \\
0 & \lambda & 0
\end{array}\right) \mathrm{e}^{-\mathrm{X} \tau}\right]=0 \\
& \left|\begin{array}{ccc}
\mathrm{X}-\left(\beta-\mu_{2}-\alpha \mathrm{T}+\mu \mathrm{Te} \mathrm{e}^{-\mathrm{X} \tau}\right) & \left(\mu_{2}+\lambda\right) \mathrm{e}^{-\mathrm{X} \tau} & 0 \\
\mu \mathrm{Te} \mathrm{e}^{-\mathrm{X} \tau}-\alpha \mathrm{T} & \mathrm{X}+\mu_{2}-\left(\mu_{2}+\lambda\right) \mathrm{e}^{-\mathrm{X} \tau} & 0 \\
0 & -\lambda \mathrm{e}^{-\mathrm{X} \tau} & \mathrm{X}+\mu_{2}
\end{array}\right|=0 \\
& {\left[\mathrm{X}^{2}+\left[\left(2 \mu_{2}-\beta+\alpha \phi\right)-(\mu+\lambda+\mu \phi) \mathrm{e}^{-\mathrm{X \tau}}\right] \mathrm{X}\right.} \\
& -\left[\mu_{2}\left(\beta-\mu_{2}-\alpha \phi\right)+\left(\mu_{2} \mu-\left(\left(\beta-\mu_{2}-\alpha \phi\right)\left(\mu_{2}+\lambda\right)\right) e^{-X \tau}+\mu \phi\left(\mu_{2}+\lambda\right) e^{-2 X \tau}\right]\right]\left[X+\mu_{2}\right] \\
& -\left[\left(\mu_{2}+\lambda\right)\left(\mu \phi \mathrm{e}^{-\mathrm{X} \tau}-\alpha \phi\right)\right]\left[\mathrm{X}+\mu_{2}\right]=0 \\
& \Rightarrow \mathrm{X}^{3}+\left[\left(2 \mu_{2}-\beta+\alpha \phi\right)-(\mu+\lambda+\mu \phi) \mathrm{e}^{-\mathrm{X} \tau}+\mu_{2}\right] \mathrm{X}^{2}+\left[\left(2 \mu_{2}^{2}-\beta \mu_{2}+\mu_{2} \alpha \phi\right)-\mu_{2}(\mu+\lambda+\mu \phi) \mathrm{e}^{-\mathrm{X} \tau}-\right. \\
& \mu 2 \beta-\mu 2-\alpha \phi-\mu 2 \mu+\beta-\mu 2-\alpha \phi \mu 2+\lambda \mathrm{e}-\mathrm{X} \tau-\mu \phi \mu 2+\lambda \mathrm{e}-2 \mathrm{X} \tau-\mu 2+\lambda \mu \phi \mathrm{e}-\mathrm{X} \tau-\alpha \phi \mathrm{e}-\mathrm{X} \tau \mathrm{X}-\mu 22 \beta-\mu 2-\alpha \phi-\mu 2 \\
& 2 \mu+\mu 2 \beta-\mu 2-\alpha \phi \mu 2+\lambda \mathrm{e}-\mathrm{X} \tau-\mu 2 \mu \phi \mu 2+\lambda \mathrm{e}-2 \mathrm{X} \tau-\mu 2 \mu 2+\lambda \mu \phi \mathrm{e}-\mathrm{X} \tau-\alpha \phi \mathrm{e}-\mathrm{X} \tau
\end{aligned}
$$

We can rewrite (5.5) thus:

$$
\begin{aligned}
& \mathrm{X}^{3}+\left(3 \mu_{2}-\beta+\alpha \phi\right) \mathrm{X}^{2}+\left[3 \mu_{2}^{2}-2 \beta \mu_{2}+2 \mu_{2} \alpha \phi-\mu_{2} \mu\right] \mathrm{X}-\mu_{2}^{2}\left(\beta-\mu_{2}-\alpha \phi\right)-\mu_{2}^{2} \mu+\left\{-(\mu+\lambda+\mu \phi) \mathrm{X}^{2}+\right. \\
& \beta-\mu 2 \mu 2+\lambda-\mu 2 \mu+\lambda+\mu \phi X+\mu 2 \beta+\alpha \phi \mu 2 \lambda-1 \mathrm{e}-\mathrm{X} \tau-\mu 2+\lambda \mu \phi(1+\mu 2) \mathrm{e}-2 \mathrm{X} \tau \text {. }
\end{aligned}
$$

Thus $\quad a_{2}=3 \mu_{2}-\beta+\alpha \phi, \quad a_{1}=3 \mu_{2}^{2}-2 \beta \mu_{2}+2 \mu_{2} \alpha \phi-\mu_{2} \mu, \quad a_{0}=-\mu_{2}^{2}\left(\beta-\mu_{2}-\alpha \phi\right)-\mu_{2}^{2} \mu$, $b_{2}=-(\mu+\lambda+\mu \phi), b_{1}=\left(\beta-\mu_{2}\right)\left(\mu_{2}+\lambda\right)-\mu_{2}(\mu+\lambda+\mu \phi) \quad, \quad b_{0}=\mu_{2} \beta+\alpha \phi \mu_{2}(\lambda-1)$ where $\phi=\frac{\mu_{2}-\beta}{\alpha-\mu}$

Analyzing the characteristic equation (5.6) at the endemic equilibrium point of the system $(2.1-2.3)$ based on Theorem 4.8 we discovered that the system is stable in the absence of delay and unstable with increasing delay if $\mu_{2}\left(\beta-\mu_{2}\right)-\beta>\alpha \phi\left(\lambda+\mu_{2}-1\right)$, that is the rate at which the birds are recruited into the system must be greater than the rate at which infection transmission is taking place.

\section{Simulation Of The Model For The Epidemiology Of Fowl Pox Infection Transmission That Incorporates Discrete Delay}

We now return to model $(2.1-2.3)$ and illustrate some of the properties discussed in the previous sections. The parameter values that we use for numerical simulations are in the table below. 


\begin{tabular}{|c|c|c|c|}
\hline Parameters & Symbol & Value & Units \\
\hline Recruitment rate & $\boldsymbol{\beta}=\mathbf{r}+\boldsymbol{\delta}$ & $\mathbf{0 . 5}$ & Birds day $^{-1}$ \\
\hline Transmission rate & $\alpha$ & $\mathbf{0 . 5 3}$ & Bird $^{-1}$ day $^{-1}$ \\
\hline Rate at which infected join the susceptible & $\boldsymbol{\mu}$ & $\mathbf{0 . 8 2}$ & Day $^{-1}$ \\
\hline Removal rate of infection & $\lambda$ & $\mathbf{0 . 7}$ & Day $^{-1}$ \\
\hline Rate of death due & $\mu_{2}$ & $\mathbf{0 . 1 2 5}$ & Day $^{-1}$ \\
\hline
\end{tabular}
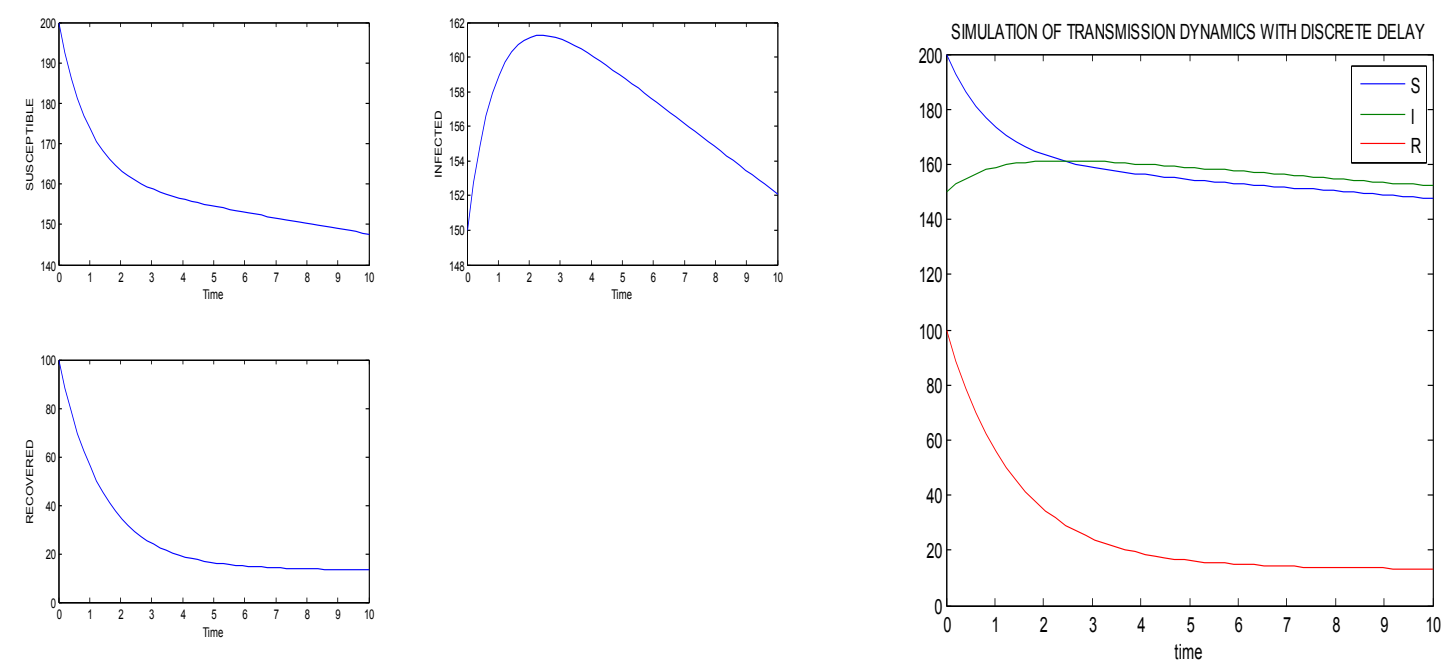

Graph of the system of transmission dynamics of fowl pox with discrete delay. As observed from the simulation, the population of the infected birds increased and become constant after a long time which agrees with the analytical analysis. While the population of the susceptible decreases and also becomes constant. Like wise the population of the removed birds. Initial conditions are $S(0)=200, I(0)=150, R(0)=100$.

\section{Summary and Conclusion}

In this paper, we have formulated the mathematical model for the epidemiology of fowl pox infection transmission that incorporates discrete delay. The model results in a discrete delay system of ordinary differential equations with delay parameter $\tau \geq 0$.

Analysing the system using theorems from differential and integral calculus Inyama, 2006, we discovered that the number of infectives after a very long time from the day of inception of the epidemic is constant. $\frac{\left(\frac{1}{2} \delta-\alpha\right) \mathrm{N}+\mu}{\frac{1}{4} \delta-\frac{1}{2} \alpha}$

The disease free equilibrium and the endemic and equilibrium of the system were both established. Using the computable criteria for stability of discrete delay system, unlike ordinary differential equations models, we obtained a exponential polynomial equations.

We also obtain the conditions for (4.7) to have negative real parts as $\beta>\mu_{2}$ and $\alpha<3 \mu$. That is the rate at which the susceptible birds are recruited into the poultry must be greater than the rate the birds die naturally. And the rate at which the birds are becoming infectious must be less than the rate at which they recover and join the susceptible population.

Hence the system is stable in the absence of delay and becomes unstable with increasing delay.Analysing the system using lemmas and theorems from, Forde, 2005, Ruan and Wei, 2001, it was established that both the disease free equilibrium and endemic equilibrium points of the system (2.1) - (2.3) are stable in the absence of delay and unstable with increasing delay if $\beta>\alpha-\mu, \mu_{2}\left(\beta-\mu_{2}\right)-\beta>$ $\alpha \phi\left(\lambda+\mu_{2}-1\right)$ that is the rate at which the birds are recruited into the system must be greater than the rate at which infection transmission is taking place.

\section{References}

[1]. Cooke, K. L, Grossman, Z., (1982), Discrete Delay, Distributed Delay and Stability Switches, Journal of Math. Anal. Appl. 86, pages $592-627,1982$

[2]. H. S. Ren,(2000), Necessary and sufficient conditions for all roots for neutral equation to have negative real parts, J. Sys. Sci. \& Math. Sci.

[3]. 20(2)(2000), 248-256. (in Chinese).

[4]. Hong-shan Ren and Hong-yan, (2002) Explicit Asymptotic Stability Criteria for Neutral Differential Equations with Two Delays , Journal of Applied Mathematics e-Notes, 2(2002), 1-9 
[5]. Ruan Shigui and Wei Junjie(2001), On the zeros of a third degree Exponential polynomial with application to a delay model for the control of testerone secretion. IMA Journal of mathematics Applied to Medicine and Biology, 18 , page 41 - 52

[6]. Obabiyi, O. S (2009), A Note on a Discrete Delay-parameter Epidemiological Model, Department of Mathematics, University of Ibadan, Nigeria

[7]. Forde ,Jonathan Erwin(2005), Delay Differential Equations Models in Mathematical Biology, Department of Mathematics University of Michigan

[8]. http://happyhenhouse.proboards43.com

[9]. Kuang, Y. (1993), Delay Differential Equations with Application Biology. Academic Press, New York,1993

[10]. Iheagwam, V. A (2006), Exponential asymptotic Stability in the Large Delay Systems, Journal of Nigerian Mathematical Physics, Vol.24, page 41-48.

[11]. Inyama, S. C. (2006) Qualitative Study of Kermack and Mckeendrick's Epidemic Model, Journal of the Nigerian Association of Mathematical Physics, Vol.10, page 477- 480. 